Research Article

\title{
Comparison of Oral Microbe Quantities from Tongue Samples and Subgingival Pockets
}

\author{
André Göhler $\mathbb{D}^{1,}{ }^{1,2}$ Stefanie Samietz, ${ }^{3}$ Carsten Oliver Schmidt $\mathbb{D}^{4},{ }^{4}$ Thomas Kocher $\mathbb{D}{ }^{5}$ \\ Ivo Steinmetz, ${ }^{1,2}$ and Birte Holtfreter $\mathbb{C}^{5}$ \\ ${ }^{1}$ Friedrich Loeffler Institute of Medical Microbiology, University Medicine Greifswald, Greifswald, Germany \\ ${ }^{2}$ Institute for Hygiene, Microbiology and Environmental Medicine, Medical University of Graz, Graz, Austria \\ ${ }^{3}$ Department of Prosthodontics, Gerostomatology and Biomaterials, University Medicine Greifswald, Greifswald, Germany \\ ${ }^{4}$ SHIP-Clinical-Epidemiological Research, Institute for Community Medicine, University Medicine Greifswald, \\ Greifswald, Germany \\ ${ }^{5}$ Department of Restorative Dentistry, Periodontology, Endodontology, and Preventive and Pediatric Dentistry, University \\ Medicine Greifswald, Greifswald, Germany
}

Correspondence should be addressed to Birte Holtfreter; birte.holtfreter@uni-greifswald.de

Received 12 October 2017; Revised 8 March 2018; Accepted 22 March 2018; Published 26 April 2018

Academic Editor: Izzet Yavuz

Copyright (C) 2018 André Göhler et al. This is an open access article distributed under the Creative Commons Attribution License, which permits unrestricted use, distribution, and reproduction in any medium, provided the original work is properly cited.

Objectives. To improve understanding of periodontitis pathology, we need more profound knowledge of relative abundances of single prokaryotic species and colonization dynamics between habitats. Thus, we quantified oral microbes from two oral habitats to gain insights into colonization variability and correlation to the clinical periodontal status. Methods. We analyzed tongue scrapings and subgingival pocket samples from 237 subjects (35-54 years) with at least 10 teeth and no recent periodontal treatment from the 11-year follow-up of the Study of Health in Pomerania. Relative abundances of Porphyromonas gingivalis, Aggregatibacter actinomycetemcomitans, Fusobacterium nucleatum, Streptococcus sanguinis, total bacteria, and Archaea were correlated to clinically assessed pocket depths (PD) and clinical attachment levels (CAL). Results. Increased relative abundances of $P$. gingivalis, A. actinomycetemcomitans, and F. nucleatum were linked to increased levels of PD and CAL (i) on the subject level (mean PD, mean $\mathrm{CAL}$ ) and (ii) in subgingival pockets. Relative abundances of Archaea from tongue samples correlated negatively with mean PD or mean CAL. Detection and quantity of bacterial species correlated weakly to moderately between the tongue and subgingival pocket, except for Archaea. Conclusions. Relative abundances of specific oral species correlated weakly to moderately between habitats. Single species, total bacteria, and Archaea were linked to clinically assessed severity of periodontitis in a habitat-dependent manner.

\section{Introduction}

Various microorganisms colonize oral habitats [1-4]. Each habitat appears to be preferentially populated by different and somewhat unique sets of microbes, whereas in periodontal disease the microbial profile in the subgingival pocket seems to narrow [5]. Periodontitis-associated microorganisms colonize not only subgingival pockets but also other habitats like the tongue dorsum. It harbors species such as Fusobacterium and Porphyromonas [6,7] and further acts as a reservoir for recolonization of periodontal pockets after periodontal therapy $[8,9]$. This raises the question whether the occurrence and proportion (relative abundance) of different taxa might affect the microbial interplay in the oral cavity. However, comparing the distribution and quantity of single prokaryotic species is complicated because of various detection and quantification methods [9-13]. In a recent study, we used qPCR for quantification of a single species [7], serving as representatives for different stages of oral biofilm formation $[9,14,15]$.

Information about Archaea in context with periodontitis is rare. According to the published literature, they were detected in periodontally diseased but not in healthy gingival sulci [16-20]. In contrast, we detected archaeal 16S rRNA 
sequences from tongue scrapings in healthy and periodontally diseased subjects in a previous study [7]. However, other recent studies showed that methanogens were enriched in chronic periodontitis and drive periodontitis due to their metabolic capacity [21, 22].

While our previous study clearly demonstrated the advantage of using bacterial relative abundance levels, it did not compare bacterial colonization patterns between the tongue and the subgingival pocket habitat, considering also the clinical periodontal status [7]. Therefore, we aimed at describing the relation between the relative abundance of four oral microbes ( $P$. gingivalis, $A$. actinomycetemcomitans, F. nucleatum, and S. sanguinis), total bacteria, and Archaea in two oral habitats (the tongue dorsum and subgingival pocket) and clinically assessed periodontitis measures in wellcharacterized study subjects from the 11-year follow-up of the population-based Study of Health in Pomerania (SHIP-2).

\section{Materials and Methods}

2.1. Study Population. The Study of Health in Pomerania (SHIP) is an ongoing longitudinal cohort study in NorthEast Germany. Baseline examinations (SHIP-0) were conducted between 1997 and 2001 with 4308 participants [23]. The 11-year follow-up (SHIP-2) included 2333 subjects (2008-2012).

For this study, we considered data of 1560 subjects, for whom data were available until the end of March 2011. Out of this collective, we selected individuals according to the following criteria: $35-54$ years of age ( $N=402$ excluded), no periodontal treatment within the last five years $(N=116$ excluded), complete dental status $(N=31$ excluded $)$, at least 10 teeth ( $N=309$ excluded), and complete information on further exclusion criteria. Pregnant women $(N=1)$, subjects with diabetes mellitus and increased hemoglobin levels (HbA1c $\geq 6.5 \%)(N=14)$, and current smokers $(N=115)$ were excluded, leaving 266 individuals. Of those, 237 individuals provided both tongue scrapings and subgingival pocket samples. The study protocol was a priori approved by the local Ethics Committee of the University of Greifswald (Registration Number BB 39/08a). This study was performed in accordance with "Strengthening the Reporting of Observational Studies in Epidemiology" guidelines for human research investigations.

2.2. Covariates. Sociodemographic and behavioral variables were assessed by computer-assisted personal interviews. School education was categorized as $<10,10$, and $>10$ years. Smoking status was defined as never, former, and current smoking. We defined diabetes mellitus as a self-reported physician's diagnosis or intake of antidiabetic medication (Anatomical Therapeutic Chemical (ATC) A10). Body height and weight were determined using calibrated scales. The body mass index (BMI) was calculated and categorized as $<25,25-<30$, and $\geq 30 \mathrm{~kg} / \mathrm{m}^{2}$.

2.3. Dental Examination. Periodontal examinations comprised probing depths (PD) and clinical attachment levels
(CAL). Measurements were assessed at four sites per tooth (disto/mid/mesiobuccal and midlingual) according to the half-mouth method, alternating on the left or right side, excluding third molars and using a periodontal probe (PCP-2, Hu-Friedy, USA). CAL was not measured if the determination of the cementoenamel junction was not clear. Teeth were counted excluding third molars. Five certified and licensed dentists conducted examinations. Calibration exercises were performed before and every 6-12 months during the course of the study. Intrarater correlations for CAL measurements were $0.67-0.89$, and interrater correlation was 0.70 .

2.4. Sample Collection and DNA Isolation. Tongue biofilm was taken from the middle third of the tongue dorsum with a sterile spatula [7]. The spatula was transferred into $2.0 \mathrm{ml}$ of phosphate-buffered saline (PBS). After shaking vigorously for $30 \mathrm{~s}$, the spatula was removed. Microbial suspensions in PBS were kept at $-80^{\circ} \mathrm{C}$ until further processing. After supragingival plaque was removed with a cotton roll, subgingival plaque was collected from the mesiobuccal pocket of the most distally located, clinically examined, upper tooth in the periodontally examined quadrants. Paper points (ISO 35; Roeka, Langenau, Germany) were inserted until the pocket base for 10 seconds. To avoid cross contamination, the sampling site was confined with cotton rolls. Paper points were stored at $-80^{\circ} \mathrm{C}$. Before DNA extraction, $230 \mu \mathrm{l}$ of lysis buffer and $20 \mu \mathrm{l}$ of proteinase K (both MagNA Pure LC DNA Isolation Kit III, Roche, Mannheim, Germany) were added and samples were incubated at $65^{\circ} \mathrm{C}$ for $10 \mathrm{~min}$ and then at $95^{\circ} \mathrm{C}$ for $10 \mathrm{~min}$. DNA was extracted as described elsewhere [7] and stored at $-20^{\circ} \mathrm{C}$.

2.5. Oligonucleotides, Plasmid Standards, and Quantitative PCR. Species- and domain-specific primers and probes (MWG-Eurofins, Ebersberg, Germany) applied in the qPCR assays are listed in Supplemental Table 1 and described in detail elsewhere [7]. Plasmid standards contained respective target sequences in a pSC-B-amp/kan vector backbone (Stratagene, La Jolla, USA). The terms "total bacteria" and "Archaea" describe the total number of detected bacterial species by using the primers/probes listed in Supplemental Table 1.

The selected oral microbes were quantified using an established qPCR assay [7]. All samples were analyzed in triplicates using a LightCycler $480-\mathrm{II}$, and $C_{\mathrm{t}}$ was calculated by $\Delta \Delta C_{\mathrm{t}}$ algorithm of the LightCycler 480 Software version 1.5.0 (Roche, Mannheim, Germany). Efficiencies between 1.9 and 2.1 ensured efficient qPCR amplification in all qPCR runs.

2.6. Statistical Analyses. Continuous data are shown as mean \pm standard deviation (SD) if normally distributed (assessed by QQ plots) or median (25\% quantile, 75\% quantile) if nonnormally distributed. Categorical data are presented as numbers (percentages).

We calculated relative abundances for $P$. gingivalis, F. nucleatum, A. actinomycetemcomitans, and S. sanguinis by 
dividing the single species count by the number of $16 \mathrm{~S}$ rRNA gene copies per sample. We knew that this method could possibly lead to an underestimation of single species relative abundances, because the estimation models for species counts within a sample are still controversially discussed $[24,25]$. The sum score equals the sum of relative abundances for three (putatively) periodontal pathogens ( $P$. gingivalis, A. actinomycetemcomitans, and $F$. nucleatum).

Relative abundance values were shifted by 1 and then log transformed (retaining distribution values of 0 ). To compare relative abundances from tongue scrapings across categories of mean $\mathrm{PD} / \mathrm{CAL}$, clinical variables were categorized (1st, 2nd +3 rd, and 4 th quartile).

To show coherence between relative abundances and periodontal status, bacterial data from tongue samples and subgingival pocket samples were correlated to subject-level periodontal data (mean PD, mean CAL) and to site-specific levels of PD and CAL of the mesiobuccal pocket. To compare relative abundances across quartile-derived categories of mean PD and mean CAL, we applied Mann-Whitney $U$ tests.

We used McNemar's test to determine differences in detection rates in tongue and subgingival pocket samples. To assess the magnitude to which reliability estimates might be biased, the Prevalence index (PI; ( $\mathrm{a}-\mathrm{d}) / N$; range -1 to 1 ) and the Bias index $(\mathrm{BI} ;(\mathrm{b}-\mathrm{c}) / N$; range 0 to 1$)$ were calculated. To circumvent problems associated with the use of kappa, the Prevalence and Bias Adjusted Kappa (PABAK) $\left(2 \cdot p_{\mathrm{o}}-1 ; p_{\mathrm{o}}\right.$, observed agreement; range -1 to +1$)$ was calculated [26] to determine the accordance in detection profiles between tongue scraping and the corresponding subgingival pocket sample (gold standard). Moreover, sensitivity and specificity with $95 \%$ confidence intervals were calculated. Relative abundances derived from tongue and subgingival pocket samples were correlated using Spearman's correlation coefficient ( $r_{\mathrm{SP}} ; 95 \%$ confidence interval). All data analyses were performed using Stata/SE 12.0 [27].

\section{Results}

3.1. Study Population. Participants were 44.1 (SD 5.5) years old, and $42.2 \%$ were male (Table 1 ). Mean PD and mean CAL were 2.41 and $1.92 \mathrm{~mm}$, respectively. The subgingival pocket sample originated from the second molar in $84.0 \%$.

3.2. Correlation of Relative Abundances on the Tongue with Clinical Periodontal Status. Overall, correlations between relative abundances from tongue samples and clinical variables were weak (Table 2). Relative abundances of $P$. gingivalis, A. actinomycetemcomitans, $F$. nucleatum, and total bacteria were significantly related to mean CAL with Spearman's correlation coefficients ranging from $r_{\mathrm{sp}}=-0.17$ (95\% CI: -0.29 to $-0.04 ; p=0.009)$ for total bacteria to $r_{\mathrm{sp}}=$ 0.26 (95\% CI: 0.14 to $0.38 ; p<0.001)$ for $P$. gingivalis. Mean PD levels were weakly correlated to $P$. gingivalis $\left(r_{\mathrm{sp}}=0.15\right.$; 95\% CI: 0.03 to $0.28 ; p=0.02)$, A. actinomycetemcomitans $\left(r_{\mathrm{sp}}=0.23\right.$; $95 \%$ CI: 0.11 to $\left.0.35 ; p<0.001\right)$, and F. nucleatum $\left(r_{\mathrm{sp}}=0.25 ; 95 \%\right.$ CI: 0.13 to $\left.0.37 ; p<0.001\right)$.
TABLE 1: Characteristics of study subjects $(N=237)$.

\begin{tabular}{|c|c|}
\hline & $\begin{array}{c}\text { Mean } \pm \text { SD or } \\
\text { number }(\%)\end{array}$ \\
\hline Age, years & $44.1 \pm 5.5$ \\
\hline Males & $100(42.2 \%)$ \\
\hline \multicolumn{2}{|l|}{ School education } \\
\hline$<10$ years & $5(2.1 \%)$ \\
\hline 10 years & $169(71.3 \%)$ \\
\hline$>10$ years & $63(26.6 \%)$ \\
\hline \multicolumn{2}{|l|}{ Smoking status } \\
\hline Never smokers & $128(54.0 \%)$ \\
\hline Former smokers & $109(46.0 \%)$ \\
\hline \multicolumn{2}{|l|}{ Body mass index, $\mathrm{kg} / \mathrm{m}^{2}$} \\
\hline$<25$ & $90(38.0 \%)$ \\
\hline $25-<30$ & $97(40.9 \%)$ \\
\hline$\geq 30$ & $50(21.1 \%)$ \\
\hline \multicolumn{2}{|l|}{$\begin{array}{l}\text { Information on periodontal } \\
\text { status on the subject level }\end{array}$} \\
\hline Bleeding on probing, $\%$ & $20.5 \pm 18.1$ \\
\hline Mean PD, mm & $2.41 \pm 0.36$ \\
\hline Percentage of sites with $\mathrm{PD} \geq 4 \mathrm{~mm}, \%$ & $8.0 \pm 10.4$ \\
\hline Mean CAL, mm & $1.92 \pm 0.91$ \\
\hline $\begin{array}{l}\text { Percentage of sites with } \\
\text { CAL }>4 \mathrm{~mm} \%\end{array}$ & $11.3 \pm 16.3$ \\
\hline Tooth count (excluding third molars) & $24.8 \pm 3.2$ \\
\hline \multicolumn{2}{|l|}{ Information on-site level } \\
\hline \multicolumn{2}{|l|}{ Tooth position } \\
\hline 31 & $2(0.8 \%)$ \\
\hline 4 & $4(1.7 \%)$ \\
\hline 5 & $10(4.2 \%)$ \\
\hline 6 & $22(9.3 \%)$ \\
\hline 7 & $199(84.0 \%)$ \\
\hline \multicolumn{2}{|l|}{$\mathrm{PD}, \mathrm{mm}(N=232)$} \\
\hline $1-2$ & $46(19.8 \%)$ \\
\hline 3 & $130(56.0 \%)$ \\
\hline$\geq 4$ & $56(24.2 \%)$ \\
\hline \multicolumn{2}{|l|}{$\mathrm{CAL}, \mathrm{mm}(N=186)$} \\
\hline $0-2$ & $100(53.8 \%)$ \\
\hline 3 & $38(20.4 \%)$ \\
\hline$\geq 4$ & $48(25.8 \%)$ \\
\hline
\end{tabular}

Data are presented as mean \pm standard deviation (SD) or numbers (percentages); PD, probing depth; CAL, clinical attachment level.

Relative abundances from tongue samples were unequally distributed across categories of mean PD and mean CAL (Table 3). Relative abundances of $P$. gingivalis $(p=0.02)$, A. actinomycetemcomitans $(p=0.0005), F$. nucleatum $(p=$ $0.0002)$, and Archaea $(p=0.04), \%$ Archaea $(p=0.006)$, and the sum score $(p=0.001)$ differed significantly between the 1st and the 4th quartiles of mean PD. Relative abundances of $P$. gingivalis $(p=0.0001), A$. actinomycetemcomitans $(p=0.005), F$. nucleatum $(p=0.005)$, and total bacteria $(p=0.04)$ and the sum score $(p=0.0001)$ differed significantly between the 1 st and the 4 th quartiles of mean CAL.

\subsection{Correlation of Relative Abundances in Pocket Samples} with Clinical Periodontal Status. For subgingival pocket samples, relative abundances correlated weakly to CAL and PD levels of respective mesiobuccal sites (Table 4). Except for S. sanguinis, all relative abundances showed weak, though 
TABLE 2: Spearman's correlation coefficients ( $r_{\mathrm{SP}} ; 95 \%$ confidence intervals) between clinical periodontal variables and relative abundances from tongue scrapings (on the subject level).

\begin{tabular}{lcc}
\hline$r_{\mathrm{SP}}(95 \% \mathrm{CI})$ & & Tongue scraping \\
& Mean CAL $(N=237)$ & $0.15(0.03,0.28)$ \\
P. gingivalis & $0.26(0.14,0.38)$ & $0.23(0.11,0.35)$ \\
A. actinomycetemcomitans & $0.24(0.12,0.36)$ & $0.25(0.13,0.37)$ \\
F. nucleatum & $0.23(0.11,0.35)$ & $-0.001(-0.13,0.13)$ \\
S. sanguinis & $-0.01(-0.14,0.11)$ & $-0.11(-0.23,0.02)$ \\
Archaea & $-0.11(-0.24,0.01)$ & $-0.15(-0.27,-0.02)$ \\
\% Archaea & $-0.10(-0.23,0.03)$ & $-0.08(-0.20,0.05)$ \\
Total bacteria & $-0.17(-0.29,-0.04)$ & $(N)$ \\
\hline
\end{tabular}

$\mathrm{PD}$, probing depth; CAL, clinical attachment level; $r_{\mathrm{SP}}$, Spearman's correlation coefficient; $95 \%$ CI, 95\% confidence interval.

significant, correlations above 0.1 with the mesiobuccal CAL (range 0.15-0.26). Mesiobuccal PD was weakly correlated to $P$. gingivalis relative abundance $\left(r_{\mathrm{sp}}=0.18 ; 95 \% \mathrm{CI}\right.$ : 0.05 to 0.30 ; $p=0.006$ ).

Consistently, the sum score differed significantly comparing pockets with a mesiobuccal PD of $\leq 2 \mathrm{~mm}$ with those with a mesiobuccal $\mathrm{PD}$ of $\geq 4 \mathrm{~mm}(p=0.03$; Figure 1$)$. Similar findings were found for detections rates $(p=0.046$; Supplemental Figure 1) and relative abundances of $P$. gingivalis $(p=0.01)$ with highest levels found in pockets with a mesiobuccal $P D \geq 4 \mathrm{~mm}$ (median relative abundance $6.3 \times 10^{-6}\left(0 ; 1.3 \times 10^{-3}\right)$; Supplemental Table 3$)$. Across categories of mesiobuccal CAL, significant differences in detection rates were found for $P$. gingivalis $(p=0.03)$ and A. actinomycetemcomitans $(p=0.046)$. Accordingly, differences in relative abundances were detected for all single species $(p<0.05)$ except $S$. sanguinis, Archaea, total bacteria, and the sum score (Supplemental Table 3).

3.4. Detection of Microorganisms in Tongue and Subgingival Pocket Samples. For P. gingivalis agreement between the tongue sample and the subgingival pocket sample was $78.5 \%$ with a moderate PABAK of 0.570 (Table 5). Among subjects with $P$. gingivalis negative subgingival pocket samples, $89.6 \%$ had a negative tongue sample (specificity). Among subjects with $P$. gingivalis positive subgingival pocket samples, $61.3 \%$ had a positive tongue sample (sensitivity). F. nucleatum was detected three times less often in tongue samples $(N=53)$ compared to subgingival pocket samples $(N=168)$. S. sanguinis was detected similarly often in both habitats with a high agreement rate $(78.5 \%)$ and a high specificity (77.7\%) and sensitivity (80.9\%). For Archaea, agreement was $32.1 \%$ and PABAK was low $(-0.359)$. Archaeal sequences were found four times more often in tongue samples $(N=173)$ compared to subgingival pocket samples $(N=40)$.

It must be mentioned that the PI and the BI were strongly deviating from the Null for A. actinomycetemcomitans $(\mathrm{PI}=$ 0.565), F. nucleatum (BI $=0.485)$, and Archaea $(\mathrm{BI}=-0.561)$, indicating some degree of bias, that would have distracted the kappa. Here, the PABAK provides an unbiased estimate of reliability.

Altogether, $16.1 \%$ of tongue scrapings but $43.1 \%$ of subgingival pocket samples contained at least two of the three (putative) periodontal pathogens ( $P$. gingivalis, $A$. actinomycetemcomitans, or F. nucleatum). Accordingly, the number of detected pathogens from tongue and subgingival pocket samples correlated weakly $\left(r_{\mathrm{sp}}=0.35\right.$; 95\% CI: 0.24 to $0.46)$.

3.5. Correlation between Relative Abundances from Tongue and Subgingival Pocket Samples. Single species and total bacterial relative abundances demonstrated weak to moderate correlations between both habitats (Table 6). Correlations were moderate for relative abundances of $P$. gingivalis $\left(r_{\mathrm{sp}}=0.63 ; 95 \% \mathrm{CI}: 0.55\right.$ to $\left.0.70 ; p<0.001\right)$ and $S$. sanguinis $\left(r_{\mathrm{sp}}=0.56 ; 95 \% \mathrm{CI}: 0.46\right.$ to $\left.0.64 ; p<0.001\right)$ and weak for relative abundances of $A$. actinomycetemcomitans $\left(r_{\mathrm{sp}}=0.16\right.$; 95\% CI: 0.03 to $0.28 ; p=0.02), F$. nucleatum $\left(r_{\mathrm{sp}}=0.21 ; 95 \%\right.$ CI: 0.09 to $0.33 ; p=0.001)$, and total bacteria $\left(r_{\mathrm{sp}}=-0.14\right.$; 95\% CI: -0.26 to $0.01 ; p=0.03)$. For illustration purposes, we present untransformed relative abundances in Supplemental Table 2. Restricting samples to those with positive relative abundances from tongue scrapings and subgingival pocket samples lead to more than halved correlation coefficients for single species (Supplemental Table 2).

\section{Discussion}

To elucidate the interplay of prokaryotic colonization in two habitats, we analyzed tongue and subgingival pocket samples from 237 participants of the 11-year follow-up of the population-based SHIP by qPCR. Relative abundances of $P$. gingivalis, $A$. actinomycetemcomitans, and $F$. nucleatum from subgingival pocket samples and tongue samples were linked to corresponding levels of PD and CAL. Quantitative inspection of oral microbes showed weak to moderate correlations in detection and quantity of oral prokaryotes between the tongue and subgingival pocket, though to different degrees depending on the species.

$P$. gingivalis is commonly regarded as periodontitis related [28, 29]. Forty-six percent of our participants were $P$. gingivalis positive on the tongue and/or in the subgingival pocket, with a significantly higher detection rate $(p=0.003)$ in the periodontal pocket $(39.2 \%)$ compared to the tongue $(30.4 \%)$. For the tongue, this rate was lower than previously described (56.7\%) [7]. Furthermore, relative abundances of $P$. gingivalis were significantly higher in subgingival pocket samples compared to tongue samples (Table 6). Further, relative abundances of $P$. gingivalis increased with increasing periodontal breakdown (Supplemental Table 3). In our 


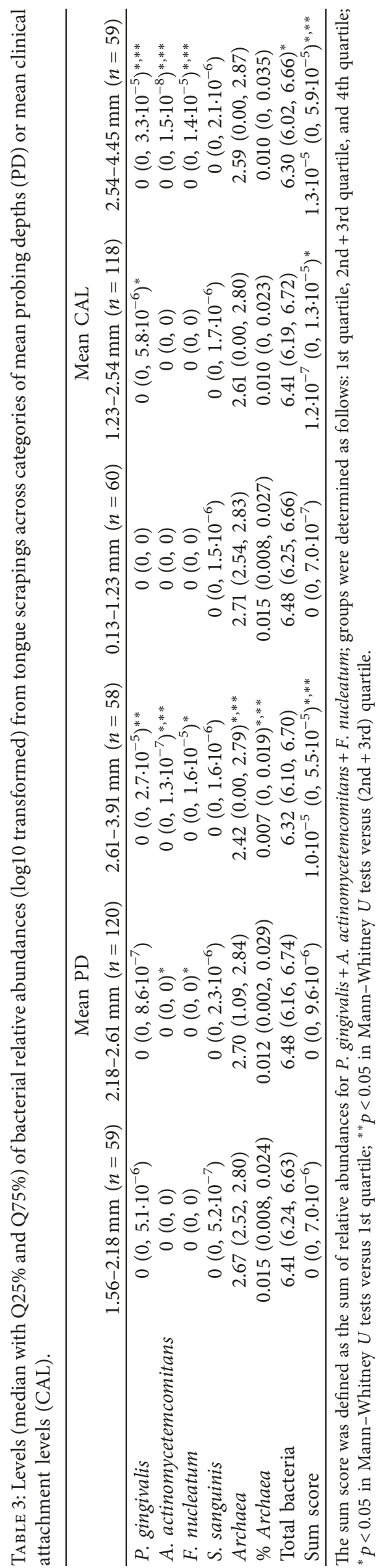


TABLE 4: Spearman's correlation coefficients ( $r_{\mathrm{SP}}$; 95\% confidence intervals) between clinical periodontal variables at the mesiobuccal site and relative abundances from subgingival pockets (site level).

\begin{tabular}{lcr}
\hline$r_{\mathrm{SP}}(95 \% \mathrm{CI})$ & Subgingival plaque \\
& Mesiobuccal CAL $(N=186)$ & Mesiobuccal PD $(N=232)$ \\
\hline P. gingivalis & $0.26(0.12,0.39)$ & $0.18(0.05,0.30)$ \\
A. actinomycetemcomitans & $0.18(0.03,0.31)$ & $0.10(-0.03,0.22)$ \\
F. nucleatum & $0.23(0.09,0.36)$ & $0.07(-0.06,0.20)$ \\
S. sanguinis & $0.07(-0.08,0.21)$ & $-0.07(-0.19,0.06)$ \\
Archaea & $0.18(0.04,0.31)$ & $0.05(-0.08,0.18)$ \\
\% Archaea & $0.17(0.02,0.30)$ & $0.04(-0.09,0.17)$ \\
Total bacteria & $0.15(0.01,0.29)$ & $0.12(-0.01,0.24)$ \\
\hline
\end{tabular}

$\mathrm{PD}$, probing depth; CAL, clinical attachment level; $r_{\mathrm{SP}}$, Spearman's correlation coefficient; $95 \%$ CI, 95\% confidence interval.

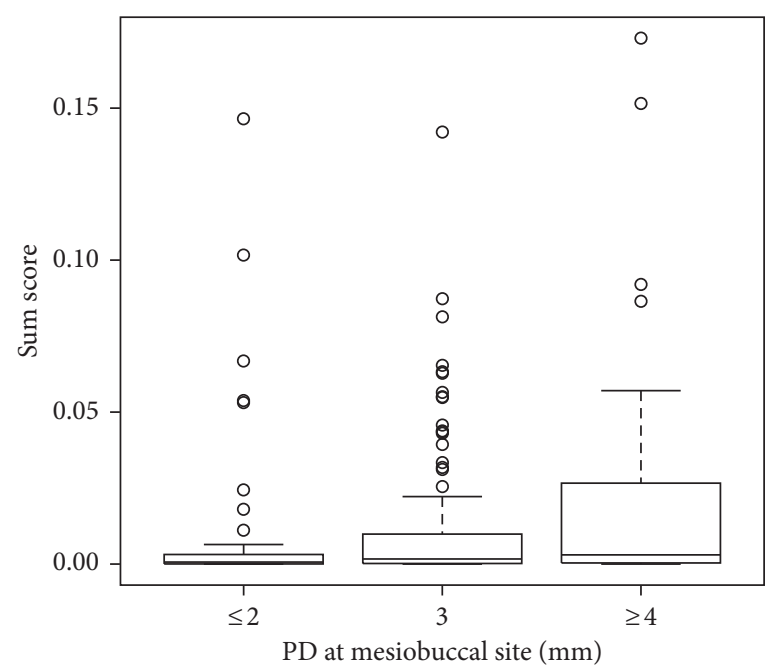

(a)

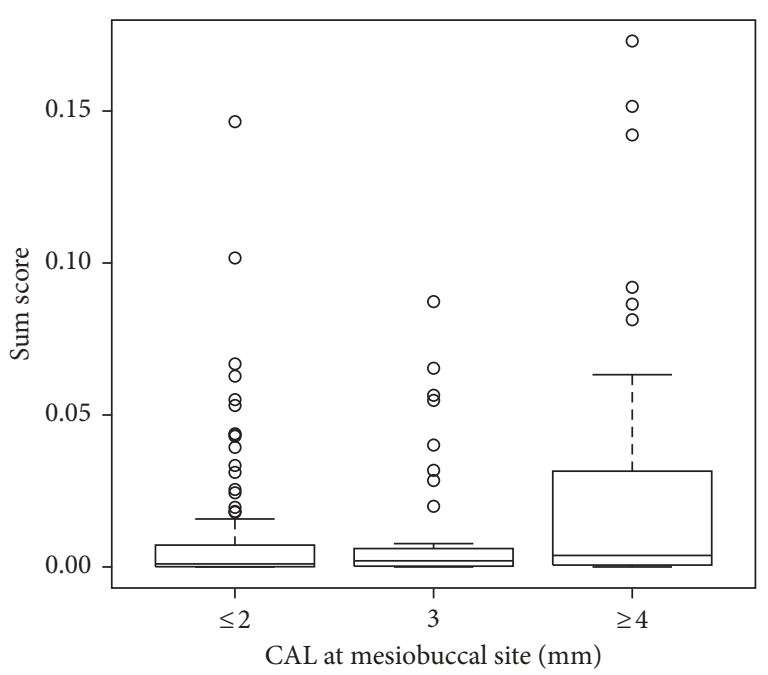

(b)

FIGURE 1: Distribution of the sum score (summed relative abundances of P. gingivalis, A. actinomycetemcomitans, and F. nucleatum) according to probing depth (PD (a)) or clinical attachment level (CAL (b)) at the same mesiobuccal sites.

study, $P$. gingivalis made up to $0.071 \%$ of total bacteria (relative abundance) in the subgingival plaque of $P$. gingivalis positive samples $(N=108)$. In addition, the strong correlation of the relative abundance between tongue and subgingival samples $\left(r_{\mathrm{SP}}=0.63 ; 95 \% \mathrm{CI}: 0.55\right.$ to 0.70$)$ confirms an interaction of these two habitats [30]. Overall, the effect of $P$. gingivalis alone was not prominent; this suggests that an ensemble of different bacteria in the respective oral habitat may determine the periodontal disease severity. However, it is possible that some taxonomic entities (sometimes referred to as "keystone pathogens") at a specific metabolic state play a more prominent role in promoting inflammation $[22,31]$.

A. actinomycetemcomitans is often found in association with periodontitis. Compared to our previous study (46.2\%) [7], the overall detection rate of $A$. actinomycetemcomitans was lower $(37.1 \%)$. One explanation may be the selection process: instead of selecting healthy and periodontally diseased pairs, we selected all subjects fulfilling the inclusion criteria. Thus, we might have selected more subjects with mild or moderate periodontitis compared to the previous study. Furthermore, the tongue was significantly $(p<0.001)$ less often colonized with A. actinomycetemcomitans (14\%) compared to the subgingival pocket (29.1\%). In Swiss adolescents, this bacterium was detected with similar rates on the tongue (approximately 20\%) and with lower rates for shallow pockets (approximately 15-17\%) [30]. In a Swedish study, similar detection rates of $30 \%$ were found in subgingival plaques of periodontitis patients [32]. In this study, relative abundances on the tongue were only weakly associated with the periodontal status, which is in contrast to findings from the previous study [7]. Taken together, these observations strengthen the hypothesis that tongue levels of A. actinomycetemcomitans are only weakly associated with severity of chronic periodontitis.

F. nucleatum, which bridges early-colonizing with latecolonizing pathogens, was found in $74.7 \%$ of all tested subjects. The detection rate was approximately three times lower in tongue samples compared to subgingival pocket samples. In line with this, we found higher relative abundances in the later ones. Relative abundances of F. nucleatum on the tongue and in the subgingival pocket correlated weakly with corresponding levels of PD and CAL. However, we might assume that members of the genus Fusobacterium belong to the core oral human microbiome $[10,33]$. The sum score was a useful marker of chronic periodontitis since 
TABLE 5: Cross table for the detection (no/yes) of the microorganisms in tongue scrapings and subgingival samples. Additionally, percentages of agreement, Prevalence and Bias Adjusted Kappa (PABAK), specificities, and sensitivities are given.

\begin{tabular}{|c|c|c|c|c|c|c|c|c|}
\hline \multirow{2}{*}{ Tongue scraping } & \multicolumn{2}{|c|}{ Subgingival sample } & \multirow{2}{*}{ Sum } & \multirow{2}{*}{$\mathrm{P}^{*}$} & \multirow{2}{*}{ Agreement } & \multirow{2}{*}{ PABAK } & \multirow{2}{*}{$\begin{array}{c}\text { Specificity } \\
(95 \% \text { CI })\end{array}$} & \multirow{2}{*}{$\begin{array}{c}\text { Sensitivity } \\
(95 \% \text { CI })\end{array}$} \\
\hline & No detection & Detection & & & & & & \\
\hline \multicolumn{9}{|l|}{ P. gingivalis } \\
\hline No detection & $129(89.6 \%)$ & $36(38.7 \%)$ & 165 & \multirow{3}{*}{$<0.001$} & \multirow{3}{*}{$78.5 \%$} & \multirow{3}{*}{0.570} & & \multirow{3}{*}{$\begin{array}{c}61.3 \% \\
(50.6 \text { to } 71.2 \%)\end{array}$} \\
\hline Detection & $15(10.4 \%)$ & $57(61.3 \%)$ & 72 & & & & $89.6 \%$ & \\
\hline Sum & 144 & 93 & 237 & & & & (83.4 to $94.1 \%)$ & \\
\hline \multicolumn{9}{|c|}{ A. actinomycetemcomitans } \\
\hline No detection & $149(88.7 \%)$ & $54(78.3 \%)$ & 203 & \multirow{3}{*}{0.04} & \multirow{3}{*}{$69.2 \%$} & \multirow{3}{*}{0.384} & & \multirow{3}{*}{$\begin{array}{c}21.7 \% \\
(12.7 \text { to } 33.3 \%)\end{array}$} \\
\hline Detection & $19(11.3 \%)$ & $15(21.7 \%)$ & 34 & & & & $\begin{array}{c}88.7 \% \\
(020)\end{array}$ & \\
\hline Sum & 168 & 69 & 237 & & & & $(82.9$ to $93.1 \%)$ & \\
\hline \multicolumn{9}{|l|}{ F. nucleatum } \\
\hline No detection & $60(87.0 \%)$ & $124(73.8 \%)$ & 184 & \multirow{3}{*}{0.03} & \multirow{3}{*}{$43.9 \%$} & \multirow{3}{*}{-0.122} & & \multirow{3}{*}{$\begin{array}{c}26.2 \% \\
(19.7 \text { to } 33.5 \%)\end{array}$} \\
\hline Detection & $9(13.0 \%)$ & $44(26.2 \%)$ & 53 & & & & $\begin{array}{l}87.0 \% \\
\end{array}$ & \\
\hline Sum & 69 & 168 & 237 & & & & (76.7 to $93.9 \%$ ) & \\
\hline \multicolumn{9}{|l|}{ S. sanguinis } \\
\hline No detection & $114(77.0 \%)$ & $17(19.1 \%)$ & 131 & \multirow{3}{*}{$<0.001$} & \multirow{3}{*}{$78.5 \%$} & \multirow{3}{*}{0.570} & & \multirow{3}{*}{$\begin{array}{c}80.9 \% \\
(71.2 \text { to } 88.5 \%)\end{array}$} \\
\hline Detection & $34(23.0 \%)$ & $72(80.9 \%)$ & 106 & & & & $\begin{array}{l}77.7 \% \\
\end{array}$ & \\
\hline Sum & 148 & 89 & 237 & & & & $(69.4$ to $83.5 \%)$ & \\
\hline \multicolumn{9}{|l|}{ Archaea } \\
\hline No detection & $50(25.4 \%)$ & $14(35.0 \%)$ & 64 & \multirow{3}{*}{0.21} & \multirow{3}{*}{$32.1 \%$} & \multirow{3}{*}{-0.359} & & \multirow{3}{*}{$\begin{array}{c}65.0 \% \\
(48.3 \text { to } 79.4 \%)\end{array}$} \\
\hline Detection & $147(74.6 \%)$ & $26(65.0 \%)$ & 173 & & & & $\begin{array}{l}25.4 \% \\
(195.321 \%\end{array}$ & \\
\hline Sum & 197 & 40 & 237 & & & & .5 to $32.1 \%)$ & \\
\hline
\end{tabular}

*McNemar test; 95\% CI, 95\% confidence interval; PABAK, Prevalence and Bias Adjusted Kappa.

TABLE 6: Overview on relative abundances (log10 transformed) for tongue scrapings and subgingival samples and respective Spearman's correlation coefficients (with $95 \%$ confidence intervals).

\begin{tabular}{lccr}
\hline & \multicolumn{2}{c}{ Relative abundances } & $r_{\mathrm{SP}}(95 \% \mathrm{CI})$ \\
\hline P. gingivalis & Tongue scraping & Subgingival sample & $0.63(0.55,0.70)$ \\
A. actinomycetemcomitans & $0\left(0,6.3 \cdot 10^{-6}\right)$ & $0\left(0,8.1 \cdot 10^{-5}\right)$ & $0.16(0.03,0.28)$ \\
F. nucleatum & $0(0,0)$ & $0\left(0,3.7 \cdot 10^{-5}\right)$ & $0.21(0.09,0.33)$ \\
S. sanguinis & $0(0,0)$ & $8.6 \cdot 10^{-4}\left(0,5.8 \cdot 10^{-3}\right)$ & $0.56(0.46,0.64)$ \\
Archaea & $0\left(0,1.6 \cdot 10^{-6}\right)$ & $0\left(0,8.9 \cdot 10^{-4}\right)$ & $-0.10(-0.23,0.03)$ \\
\% Archaea & $2.63(0,2.82)$ & $0(0,0)$ & $-0.01(-0.14,0.12)$ \\
Total bacteria & $0.011(0,0.025)$ & $0(0,0)$ & $-0.14(-0.26,0.01)$ \\
\hline
\end{tabular}

Relative abundances are given as median (Q25\%, Q75\%); $r_{\mathrm{SP}}$, Spearman's correlation coefficient; 95\% CI, 95\% confidence interval.

higher levels correlated with higher levels of PD and CAL in both habitats (Figure 1).

S. sanguinis frequently colonizes in the healthy human mouth $[34,35]$. In contrast to another study in which S. sanguinis was not detected on the tongue dorsum of any of the five examined healthy subjects [3], we detected $S$. sanguinis on the middle third of the tongue dorsum in $44.7 \%$ of subjects. However, this rate was lower compared to the detection rate of $57.4 \%$ in our previous study [7]. In subgingival pocket samples, we detected $S$. sanguinis in $37.5 \%$ of samples. This value was between the reported carriage percentages of $25 \%$ (periodontally diseased) and $40 \%$ (healthy) of a Japanese study [33]. Moreover, the proportion of $S$. sanguinis in positive subgingival plaques was $0.37 \%$, which was in line with a previous study reporting a proportion of $0.33 \% \pm 0.57$ for $S$. sanguinis in healthy subjects [33].

Members of the Archaea also colonize the oral cavity, especially within subgingival plaques and on tooth surfaces
[21, 36-38], while the diversity seems to be very narrow. We detected archaeal sequences in $73.0 \%$ of tongue samples, but only $16.9 \%$ of subgingival pocket samples. The latter proportion was lower than the so far reported detection range for subgingival pocket samples of $36 \%$ [39] to $96.4 \%$ [36]. However, the archaeal abundance is probably higher in periodontal pockets with $>5 \mathrm{~mm}$ pocket depth [21], linking the archaeal abundance to more severe periodontal disease. In this study, we did evaluate a relation between archaeal load and severe probing depths, because the number of subjects with mesiobuccal $\mathrm{PD}>5 \mathrm{~mm}$ was only seven, precluding further analyses.

Further, Matarazzo et al. found strong correlations between $P$. gingivalis and the archaeal count $(r=0.75)$ in subjects with chronic periodontitis [40]. We found moderate correlations between total bacterial and archaeal load on the tongue ( $r_{\text {sp }}=0.53 ; 95 \%$ CI: 0.44 to 0.62$)$ and within the subgingival plaque $\left(r_{\mathrm{sp}}=0.41 ; 95 \%\right.$ CI: 0.30 to 0.51$)$. The relative abundance of $F$. nucleatum was only weakly related 
to the archaeal relative abundance in subgingival plaque $\left(r_{\mathrm{sp}}=0.14 ; 95 \%\right.$ CI: 0.02 to 0.27$)$. Thus, our study only slightly supports the hypothesis of Matarazzo et al. that Archaea may favor the settlement of some anaerobic bacterial species such as $P$. gingivalis, F. nucleatum, or Prevotella intermedia [21, 40]. Very weak and nonsignificant correlations were found between relative abundances of $P$. gingivalis or A. actinomycetemcomitans and archaeal load in both habitats $\left(-0.08<r_{\mathrm{SP}}<0.09\right)$ showing that not all virulent species are related to the archaeal load. Additionally, archaeal relative abundances on the dorsal tip of the tongue and the subgingival pocket did not correlate $\left(r_{\mathrm{SP}}=-0.10 ; 95 \% \mathrm{CI}\right.$ : -0.23 to 0.03$)$. This, together with the previous observation that low archaeal relative abundances correlate with healthassociated effects [7] indicates a more complex picture. Further, metagenome analysis might reveal distinct bacterial/archaeal patterns, which dominate in each habitat depending on the periodontal condition.

For this study a well-defined, homogenous, and periodontally untreated subgroup was a priori selected to ensure that associations seen between tongue scrapings, pocket samples, and clinical periodontal status are more likely to be unbiased by subject-related factors. Specifically, with regard to disease severity, study subjects cover the range from periodontally healthy to moderately diseased subjects, reflecting the general population in contrast to clinical patient cohorts. Some limitations deserve consideration. First, periodontal examinations were taken according to the half-mouth method at four sites per tooth, which might have led to an underestimation of periodontal disease severity on the subject level [41]. Second, an aspect common to most sampling methods applied in oral habitats is that cross contamination of samples cannot be completely excluded.

\section{Conclusions}

In this study, relative abundances of specific periopathogenic bacteria sampled from subgingival pocket samples and tongue samples correlated significantly to corresponding levels of PD and CAL. Further, we indicated relevant correlations for four periodontal bacteria and Archaea between a global oral habitat (the tongue) and a local site (the subgingival pocket sample). This strengthens the hypothesis that the tongue could serve as a reservoir for oral bacteria. In consequence, considering the relation between both habitats might be helpful during/after dental cleaning-events or reinfections. Moreover, the detection and quantification of tongue microbes might help to determine the risk of recolonization of debrided sites after periodontal treatment. Further, the strong correlations between $P$. gingivalis and periodontal parameter variables point to its usage as an indicator for periodontal disease severity.

\section{Ethical Approval}

All procedures performed in studies involving human participants were in accordance with the ethical standards of the institutional and/or national research committee and with the 1964 Helsinki Declaration and its later amendments or comparable ethical standards.

\section{Consent}

Informed consent was obtained from all study participants.

\section{Conflicts of Interest}

The authors declare that they have no conflicts of interest.

\section{Acknowledgments}

SHIP is part of the Community Medicine Research net (CMR) of the University of Greifswald, Germany, which is funded by the Federal Ministry of Education and Research under Grant nos. ZZ9603, 01ZZ0103, and 01ZZ0701 and the Ministry of Cultural Affairs as well as the Social Ministry of the Federal State of Mecklenburg-West Pomerania (http://www. community-medicine.de). Microbial analysis and André Göhler were supported by the Federal Ministry of Education and Research under Grant no. 03138010. The authors acknowledge the SHIP data collection team, which comprises dental and medical examiners, technicians, interviewers, and assistants. The authors also thank Anne Kohler and Claudia Wiede for their technical assistance.

\section{Supplementary Materials}

Supplemental Table 1: sequence information, specificity, amplicon size, and molarity of oligonucleotide primers and probes used in the qPCR assays. Supplemental Table 2: correlation of abundances between tongue scrapings and subgingival samples. Supplemental Table 3: median relative abundances ( $\log 10$ transformed; with $25 \%$ and $75 \%$ quantiles) from subgingival pocket samples according to categories of mesiobuccal probing depths and clinical attachment levels (categorized as $\leq 2,3$, and $\geq 4 \mathrm{~mm}$ ). Supplemental Figure 1: detection rates of bacteria in subgingival plaques according to (a) mesiobuccal probing depth (PD) or to (b) mesiobuccal clinical attachment level (CAL). $P$ values were calculated from chi-squared tests evaluating differences in detection rates among groups. (Supplementary Materials)

\section{References}

[1] B. J. Keijser, E. Zaura, S. M. Huse et al., "Pyrosequencing analysis of the oral microflora of healthy adults," Journal of Dental Research, vol. 87, no. 11, pp. 1016-1020, 2008.

[2] C. Huttenhower, D. Gevers, R. Knight et al., "Structure, function and diversity of the healthy human microbiome," Nature, vol. 486, no. 7402, pp. 207-214, 2012.

[3] J. A. Aas, B. J. Paster, L. N. Stokes, I. Olsen, and F. E. Dewhirst, "Defining the normal bacterial flora of the oral cavity," Journal of Clinical Microbiology, vol. 43, no. 11, pp. 5721-5732, 2005.

[4] D. L. Mager, L. A. Ximenez-Fyvie, A. D. Haffajee, and S. S. Socransky, "Distribution of selected bacterial species on intraoral surfaces," Journal of Clinical Periodontology, vol. 30, no. 7, pp. 644-654, 2003. 
[5] B. Liu, L. L. Faller, N. Klitgord et al., "Deep sequencing of the oral microbiome reveals signatures of periodontal disease," PLoS One, vol. 7, no. 6, p. e37919, 2012.

[6] A. M. Eren, G. G. Borisy, S. M. Huse, and J. L. Mark Welch, "Oligotyping analysis of the human oral microbiome," Proceedings of the National Academy of Sciences, vol. 111, no. 28, pp. E2875-E2884, 2014.

[7] A. Gohler, A. Hetzer, B. Holtfreter et al., "Quantitative molecular detection of putative periodontal pathogens in clinically healthy and periodontally diseased subjects," PLoS One, vol. 9, no. 7, p. e99244, 2014.

[8] A. C. R. Tanner, B. J. Paster, S. C. Lu et al., "Subgingival and tongue microbiota during early periodontitis," Journal of Dental Research, vol. 85, no. 4, pp. 318-323, 2006.

[9] C. Do Nascimento, J. P. M. Issa, E. Watanabe, and I. Y. Ito, "DNA checkerboard method for bacterial detection of microbiota from teeth and tongue biofilms. A preliminary study," Minerva Stomatologica, vol. 57, no. 11-12, pp. 561-567, 2008.

[10] K. Boutaga, A. J. van Winkelhoff, C. M. J. E. VandenbrouckeGrauls, and P. H. M. Savelkoul, "The additional value of real-time PCR in the quantitative detection of periodontal pathogens," Journal of Clinical Periodontology, vol. 33, no. 6, pp. 427-433, 2006.

[11] B. J. Paster, I. Olsen, J. A. Aas, and F. E. Dewhirst, "The breadth of bacterial diversity in the human periodontal pocket and other oral sites," Periodontology 2000, vol. 42, no. 1, pp. 80-87, 2006.

[12] S. Yang, S. Lin, G. D. Kelen et al., "Quantitative multiprobe PCR assay for simultaneous detection and identification to species level of bacterial pathogens," Journal of Clinical Microbiology, vol. 40, no. 9, pp. 3449-3454, 2002.

[13] H. J. Lee, J. K. Kim, J. Y. Cho, J. M. Lee, and S. H. Hong, "Quantification of subgingival bacterial pathogens at different stages of periodontal diseases," Current Microbiology, vol. 65, no. 1, pp. 22-27, 2012.

[14] P. E. Kolenbrander, R. N. Andersen, D. S. Blehert, P. G. Egland, J. S. Foster, and R. J. Palmer, "Communication among oral bacteria," Microbiology and Molecular Biology Reviews, vol. 66, no. 3, pp. 486-505, 2002.

[15] J. M. ten Cate, "Biofilms, a new approach to the microbiology of dental plaque," Odontology, vol. 94, no. 1, pp. 1-9, 2006.

[16] N. Belay, B. S. Rajagopal, and L. Daniels, "Methanogenic bacteria from human dental plaque," Applied and Environmental Microbiology, vol. 54, no. 2, pp. 600-603, 1988.

[17] C. L. Li, D. L. Liu, Y. T. Jiang et al., "Prevalence and molecular diversity of Archaea in subgingival pockets of periodontitis patients," Oral Microbiology and Immunology, vol. 24, no. 4, pp. 343-346, 2009.

[18] E. M. Kulik, "Identification of archaeal rDNA from subgingival dental plaque by PCR amplification and sequence analysis," FEMS Microbiology Letters, vol. 196, no. 2, pp. 129-133, 2001.

[19] P. B. Eckburg, P. W. Lepp, and D. A. Relman, “Archaea and their potential role in human disease," Infection and Immunity, vol. 71, no. 2, pp. 591-596, 2003.

[20] K. Yamabe, H. Maeda, S. Kokeguchi et al., "Distribution of Archaea in Japanese patients with periodontitis and humoral immune response to the components," FEMS Microbiology Letters, vol. 287, no. 1, pp. 69-75, 2008.

[21] H. P. Horz, N. Robertz, M. E. Vianna, K. Henne, and G. Conrads, "Relationship between methanogenic archaea and subgingival microbial complexes in human periodontitis," Anaerobe, vol. 35, pp. 10-12, 2015.
[22] Z. L. Deng, S. P. Szafrański, M. Jarek, S. Bhuju, and I. WagnerDöbler, "Dysbiosis in chronic periodontitis: key microbial players and interactions with the human host," Scientific Reports, vol. 7, no. 1, p. 3703, 2017.

[23] U. John, E. Hensel, J. Lüdemann et al., "Study of Health in Pomerania (SHIP): a health examination survey in an east German region: objectives and design," Sozial- und Praventivmedizin, vol. 46, no. 3, pp. 186-194, 2001.

[24] J. Bunge, S. S. Epstein, and D. G. Peterson, "Comment on "Computational improvements reveal great bacterial diversity and high metal toxicity in soil"," Science, vol. 313, no. 5789, p. 918, 2006.

[25] I. Volkov, J. R. Banavar, and A. Maritan, "Comment on "Computational improvements reveal great bacterial diversity and high metal toxicity in soil"," Science, vol. 313, no. 5789, p. 918, 2006.

[26] T. Byrt, J. Bishop, and J. B. Carlin, "Bias, prevalence and kappa," Journal of Clinical Epidemiology, vol. 46, no. 5, pp. 423-429, 1993.

[27] StataCorp, Stata Statistical Software: Release 12, StataCorp LP, College Station, TX, USA, 2011.

[28] B. J. Paster, S. K. Boches, J. L. Galvin et al., "Bacterial diversity in human subgingival plaque," Journal of Bacteriology, vol. 183 , no. 12 , pp. $3770-3783,2001$.

[29] J. Slots, "Subgingival microflora and periodontal disease," Journal of Clinical Periodontology, vol. 6, no. 5, pp. 351-382, 1979.

[30] S. Eick, M. Pietkiewicz, and A. Sculean, "Oral microbiota in Swiss adolescents," Clinical Oral Investigations, vol. 17, no. 1, pp. 79-86, 2013.

[31] G. Hajishengallis, R. P. Darveau, and M. A. Curtis, "The keystone-pathogen hypothesis," Nature Reviews Microbiology, vol. 10, no. 10, pp. 717-725, 2012.

[32] R. Claesson, C. Höglund-Åberg, D. Haubek, and A. Johansson, "Age-related prevalence and characteristics of Aggregatibacter actinomycetemcomitans in periodontitis patients living in Sweden," Journal of Oral Microbiology, vol. 9, no. 1, p. 1334504, 2017.

[33] Y. Abiko, T. Sato, G. Mayanagi, and N. Takahashi, "Profiling of subgingival plaque biofilm microflora from periodontally healthy subjects and from subjects with periodontitis using quantitative real-time PCR," Journal of Periodontal Research, vol. 45, no. 3, pp. 389-395, 2010.

[34] P. Belda-Ferre, L. D. Alcaraz, R. Cabrera-Rubio et al., "The oral metagenome in health and disease," ISME Journal, vol. 6, no. 1, pp. 46-56, 2012.

[35] J. Kreth, R. A. Giacaman, R. Raghavan, and J. Merritt, “The road less traveled-defining molecular commensalism with Streptococcus sanguinis," Molecular Oral Microbiology, vol. 32, no. 3, pp. 181-196, 2017.

[36] F. Matarazzo, A. C. Ribeiro, M. Feres, M. Faveri, and M. P. Alves Mayer, "Diversity and quantitative analysis of Archaea in aggressive periodontitis and periodontally healthy subjects," Journal of Clinical Periodontology, vol. 38, no. 7, pp. 621-627, 2011.

[37] B. Dridi, D. Raoult, and M. Drancourt, "Archaea as emerging organisms in complex human microbiomes," Anaerobe, vol. 17, no. 2, pp. 56-63, 2011.

[38] M. Efenberger, J. Agier, E. Pawłowska, and E. BrzezińskaBłaszczyk, "Archaea prevalence in inflamed pulp tissues," Central European Journal of Immunology, vol. 40, no. 2, pp. 194-200, 2015.

[39] P. W. Lepp, M. M. Brinig, C. C. Ouverney, K. Palm, G. C. Armitage, and D. A. Relman, "Methanogenic Archaea 
and human periodontal disease," Proceedings of the National Academy of Sciences of the United States of America, vol. 101, no. 16, pp. 6176-6181, 2004.

[40] F. Matarazzo, A. C. Ribeiro, M. Faveri, C. Taddei, M. B. Martinez, and M. P. A. Mayer, "The domain Archaea in human mucosal surfaces," Clinical Microbiology and Infection, vol. 18, no. 9, pp. 834-840, 2012.

[41] A. Kingman, C. Susin, and J. M. Albandar, "Effect of partial recording protocols on severity estimates of periodontal disease," Journal of Clinical Periodontology, vol. 35, no. 8, pp. 659-667, 2008. 


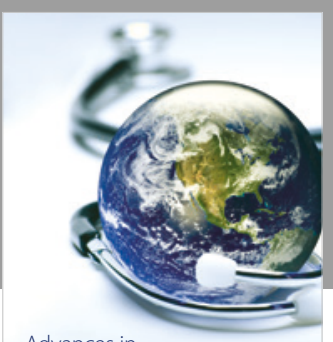

Advances in
Public Health

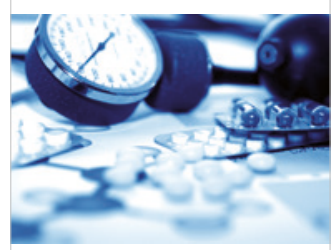

Case Reports in

Medicine

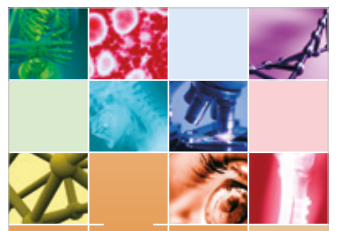

niernational Journal of

Biomaterials
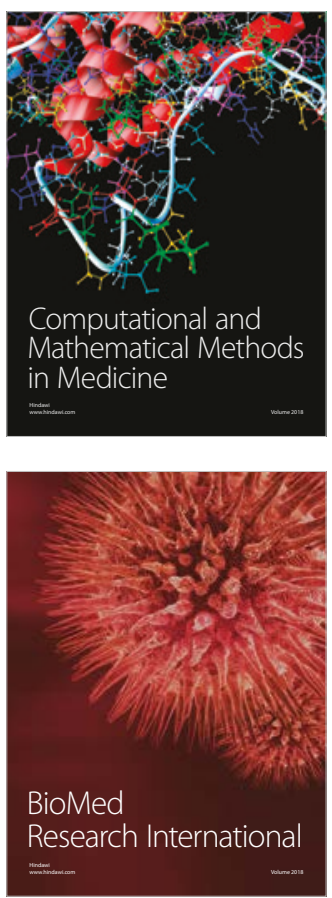

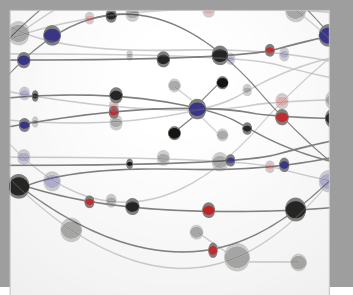

The Scientific World Journal Dentistry

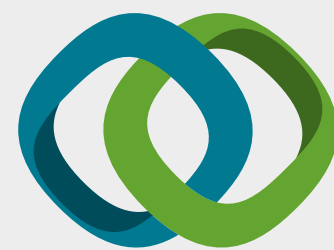

Hindawi

Submit your manuscripts at

www.hindawi.com
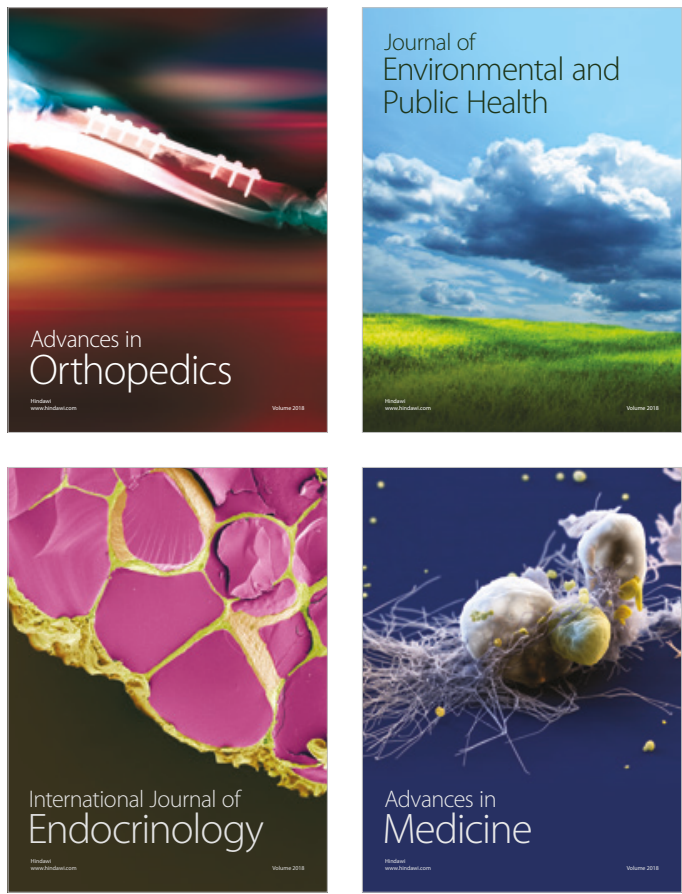
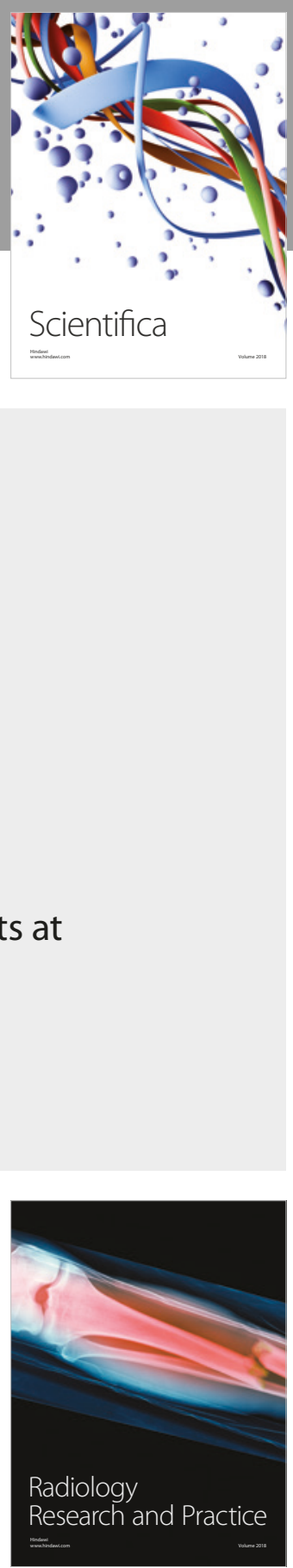

Scientifica

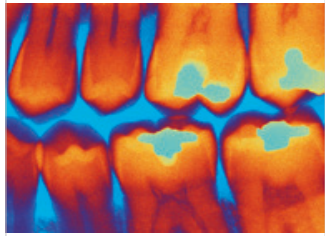

Case Reports in

Dentistry
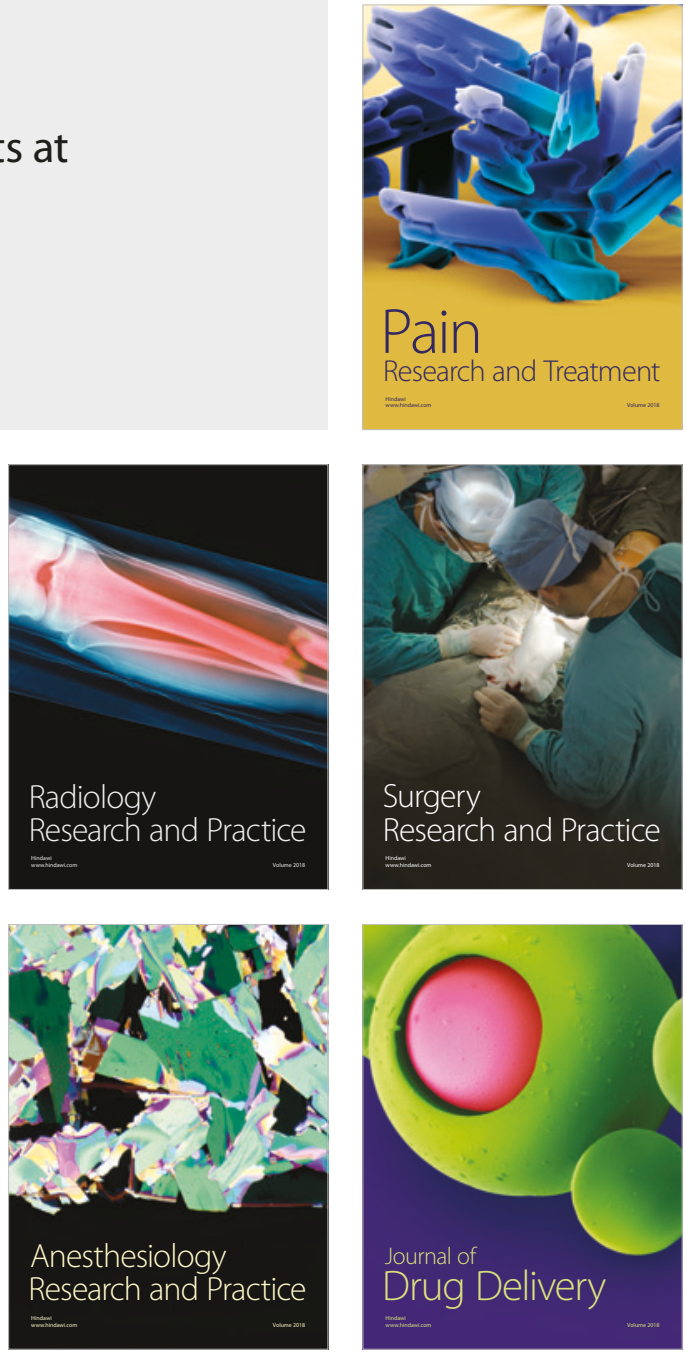